\title{
Is continuous insulin treatment safe in aneurysmal subarachnoid hemorrhage?
}

\author{
Florian Schlenk \\ Asita S Sarrafzadeh \\ Clinic of Neurosurgery, Charité \\ Campus Virchow Medical Center, \\ Berlin, Germany
}

Correspondence: Asita S Sarrafzadeh Clinic of Neurosurgery, Charité Virchow Medical Center, Charité Universitätsmedizin Berlin, Augustenburger Platz I, 13353, Berlin, Germany

Tel +4930450660264

Fax +49 30450560900

Email asita.sarrafzadeh@charite.de
Objectives: To investigate the long-term effect of continuous insulin infusion for glucose control on cerebral metabolism in aneurysmal subarachnoid hemorrhage (SAH) patients.

Methods: Prospective, nonrandomized study of 31 SAH patients in the ICU (52 \pm 10 years, WFNS Grade $2.9 \pm 1.6$ ). A microdialysis catheter was inserted into the vascular territory of the aneurysm. Metabolic changes during 4 days after onset of insulin infusion were analyzed. Blood glucose levels $>140 \mathrm{mg} / \mathrm{dL}$ after clinical stabilization were treated with intravenous insulin.

Results: 24 patients were treated with intravenous insulin. Though no insulin-induced hypoglycemia occurred, cerebral glucose decreased on days 1-4 after insulin onset without reaching critical levels. Glycerol, a marker of membrane degradation, showed a reversible increase on day 1 while the lactate/pyruvate ratio remained stable and glutamate even decreased indicating absence of severe cerebral crisis following insulin infusion and excluding ischemia as a cause for cerebral glucose depletion.

Conclusions: Concerning cerebral metabolism, long-term continuous insulin infusion appears to be safe as long as cerebral glucose levels do not fall below the physiological range. In view of the high incidence of hyperglycemia and need for insulin treatment, future studies on the effect of insulin on cerebral metabolism in SAH patients are desirable.

Keywords: glucose, hyperglycemia, insulin, subarachnoid hemorrhage, microdialysis

\section{Introduction}

Hyperglycemia has been shown to increase infarct volume and neurological deficits in many experimental models of cerebral hypoxic-ischemic injury (Ginsberg et al 1980; D'Alecy et al 1986). Clinical studies also indicate an adverse effect on outcome when cerebral ischemia occurs in the setting of hyperglycemia (Pulsinelli et al 1983). In acute stroke and aneurysmal subarachnoid hemorrhage ( $\mathrm{SAH})$, mortality rates are increased if fasting plasma glucose concentrations exceed $6.1 \mathrm{mmol} / \mathrm{L}(110 \mathrm{mg} / \mathrm{dl})(\mathrm{Candelise}$ et al 1985; Badjatia et al 2005; Frontera et al 2006). These findings suggest that even mild hyperglycemia, induced both exogenously or endogenously, significantly impairs neurological post-ischemic outcome.

Cerebral microdialysis (MD), an established neuromonitoring technique analyzing cerebral extracellular fluid, can measures glucose, the main source of energy to the brain, and its metabolites online in the neurointensive care unit (Ungerstedt 1991; Hutchinson et al 2002; Sarrafzadeh et al 2002). In traumatic brain injury (TBI) patients, low cerebral glucose was associated with unfavorable outcome (Vespa et al 2003), and in SAH with a severe metabolic derangement (Schlenk et al 2008b). Targeted insulin therapy for glycemic control induced a decrease in cerebral glucose and an increase in markers of cerebral ischemia in TBI patients (Vespa et al 2006). In SAH patients, insulin treatment did not show evidence of clinical deterioration (Bell et al 2005), but induced mild cerebral glucose depletion in the first hours of infusion (Schlenk et al 2008a). However, lacking respective data on the long-term effects of insulin on cerebral 
metabolism in SAH patients, the purpose of this study was to investigate the potentially harmful effect of insulin treatment on cerebral metabolism in SAH patients.

\section{Clinical material and methods}

\section{Patient characteristics and management}

This study was approved by the Local Research Ethics Committee at Charité Virchow Medical Center, in accordance with the Declaration of Helsinki as revised in Edinburgh in October 2000, and is part of an ongoing prospective study on cerebral metabolism monitored by bedside microdialysis in aneurysmal SAH patients.

Thirty-one consecutive patients were enrolled who were admitted to the neurosurgical department of a university hospital between August 2005 and June 2007 and met the following criteria: 1) SAH confirmed by head computerized tomography (CT); 2) cerebral angiogram demonstrating intracranial aneurysm(s); 3) surgical therapy or endovascular therapy in combination with ventricular drainage. Exclusion criteria were hemodynamic instability, fixed and dilated pupils on admission, death within $24 \mathrm{hrs}$ after admission and endovascular therapy of the posterior cerebral circulation.

All patients considered surgical candidates were managed according to a uniform protocol detailed previously (Sarrafzadeh et al 2002). Depending on their neurological course, the patients were classified as asymptomatic patients $(\mathrm{n}=6)$ and patients presenting acute focal (AFND, $\mathrm{n}=14)$ or delayed ischemic neurological deficits (DIND, $\mathrm{n}=11$ ), with detailed definitions described previously (Sarrafzadeh et al 2002). In case of any neurological deterioration, a CT was performed to rule out hydrocephalus, infarction, or rebleeding/intracerebral hemorrhage as possible causes of deterioration.

Glucose levels were targeted to be $<7.8 \mathrm{mmol} / \mathrm{L}$ $(<140 \mathrm{mg} / \mathrm{dL})$ using continuous intravenous regular insulin if necessary. Routine glycemic control consisted of blood glucose checks every $4 \mathrm{hrs}$ (6 hrs in patients without insulin treatment) and insulin infusion starting at a flow rate of $2 \mathrm{ml} / \mathrm{h}$ (with $1 \mathrm{ml}=1 \mathrm{IE}$ ) and adjusted by the bedside nurse according to the actual blood glucose level (maximum $8 \mathrm{ml} / \mathrm{h}$ ). In patients with instable glucose levels, blood glucose was checked every 1 to $3 \mathrm{hrs}$. Global handicap was assessed using the Glasgow Outcome Scale (GOS) at 3 months (Jennett et al 1975).

\section{Bedside microdialysis}

A microdialysis catheter (CMA 70, CMA Microdialysis, Solna, Sweden; length $10 \mathrm{~mm}$, molecular weight limit of 100.000 Dalton) was inserted immediately after aneurysm clipping or with placement of a ventricular drainage into brain parenchyma of the corresponding vascular territory of the aneurysm, eg, the right frontal lobe in patients with an anterior communicating artery aneurysm. Care was taken to avoid insertion into macroscopically lesioned brain tissue or into an intracerebral hemorrhage. Catheters were perfused with sterile Ringer's solution at a flow rate of $0.3 \mu \mathrm{L} / \mathrm{min}$. On the outlet tube perfusates were collected in microvials, exchanged hourly and analyzed immediately at bedside in a mobile photometric, enzyme-kinetic analyzer (CMA 600, CMA Microdialysis, Solna, Sweden). The estimated recovery for the system is $0.65-0.72$ (Hutchinson et al 2000b). Parameters of energy metabolism (glucose, pyruvate, lactate, lactate/pyruvate ratio), glycerol (a marker of membrane degradation) and glutamate (a marker of ischemia) were analyzed (Ungerstedt 1991; Saveland et al 1996; Hutchinson et al 2000a). MD data are presented as daily medians calculated from hourly measured microdialysate concentrations. A critical decrease in cerebral glucose was defined as a decrease below $0.6 \mathrm{mmol} / \mathrm{L}$, corresponding to the mean level minus 1 standard deviation of our SAH patients without any neurological symptoms (Schlenk et al 2008b).

\section{Data analysis}

All data were collected during days 1-10 after admission due to SAH. Between-group comparisons were performed using Kruskal-Wallis one-way analysis of variance. Statistical analysis of not normally distributed sequential data over time was performed using Wilcoxon signed-rank test. Data in the tables and text are expressed as mean \pm standard deviation if not specified otherwise. Differences were considered statistically significant at $p<0.05$. All statistics were done using SPSS 14.0 (SPSS Inc., Chicago, IL, USA).

\section{Results}

Demographic and clinical characteristics of the 31 patients are summarized in Table 1. Patients were classified into a noninsulin-treatment group $(\mathrm{n}=7)$ and an insulin-treatment group $(\mathrm{n}=24)($ Table 1$)$.

\section{Blood glucose and insulin treatment}

After stabilization on day 1 after SAH, insulin treatment was necessary in 24 patients. Median blood glucose on the day of onset of insulin infusion was $7.9 \pm 0.8 \mathrm{mmol} / \mathrm{L}$ (141.5 \pm $14.7 \mathrm{mg} / \mathrm{dL}$ ). No insulin-induced hypoglycemia occurred within 10 days after SAH.

Cerebral vasospasm as an insulin-independent cause for the observed glucose depletion can widely be excluded as 
Table I Characteristics of 3 I SAH patients. Data are expressed as mean \pm SD or absolute numbers and percentage, $p$ values are given for between-group comparison of insulin-treated and not insulin-treated patients (Kruskal-Wallis one-way analysis of variance)

\begin{tabular}{|c|c|c|c|}
\hline & \multicolumn{2}{|l|}{ Treatment groups } & \multirow[t]{2}{*}{$\mathbf{p}$} \\
\hline & Insulin treatment $(n=24)$ & No insulin treatment $(n=7)$ & \\
\hline Age (years) & $54.0 \pm 9.5$ & $46.0 \pm 8.7$ & 0.04 \\
\hline Gender: male/female & $7 / 17$ & $1 / 6$ & 0.44 \\
\hline \multicolumn{4}{|l|}{ Neurological symptoms: } \\
\hline Asymptomatic/AFND/DIND & $1 / 14 / 9$ & $5 / 0 / 2$ & 0.03 \\
\hline WFNS score & $3.3 \pm 1.6$ & $1.6 \pm 0.8$ & 0.02 \\
\hline I (\%), II (\%), III (\%) & $5(2 I), 4(17), 2(8)$ & 4 (57), 2 (29), I (14) & \\
\hline IV (\%),V (\%) & 6 (25), 7 (29) & 0,0 & \\
\hline Fisher score & $3.7 \pm 0.6$ & $3.2 \pm 0.8$ & 0.06 \\
\hline Time SAH - surgery (hrs) & $14.5 \pm 7.8$ & $15.4 \pm 7.9$ & 0.72 \\
\hline GOS after 3 months & $3.13 \pm 1.62$ & $4.83 \pm 0.41$ & 0.02 \\
\hline
\end{tabular}

Abbreviations:WFNS,World Federation of Neurological Surgeons Grading of SAH (Drake 1988); AFND, acute focal neurological deficit; DIND, delayed ischemic neurological deficit; SAH, subarachnoid hemorrhage; GOS, Glasgow Outcome Scale (Jennett et al 1975).

insulin infusion started before onset of DIND in almost all DIND patients.

\section{Long-term effect of insulin on cerebral metabolism}

As recently a decrease in cerebral glucose and a trend to elevated glycerol levels within the first 12 hours after onset of insulin infusion were observed, we performed this analysis to study the long-term effects of insulin (Schlenk et al 2008a). Microdialysis parameters were analyzed daily for 4 days after onset of insulin infusion. Blood glucose remained stable after insulin onset, and no hypoglycemia occurred. Cerebral glucose decreased on days 1 to 4 after insulin onset, however it did not reach critically low levels ( $\mathrm{p}=0.009$; Figure 1).

Glycerol, a marker of cellular membrane degradation, increased until day 1 after insulin onset $(p=0.049)$ and then decreased again reaching baseline levels on day 4 after onset of insulin infusion (Figure 2A). The lactate/pyruvate ratio (LPR), a marker of anaerobic metabolism, remained stable indicating absence of cellular distress (Figure 2B). Interestingly, extracellular concentrations of glutamate decreased during insulin infusion $(\mathrm{p}<0.05)$, indicating that no ischemia or relevant neuronal damage was present during the observation period (Figure 2C).

\section{Discussion}

There are two principal findings of this study. First, though no insulin-induced hypoglycemia occurred, cerebral glucose decreased on days 1-4 after insulin onset, however without reaching critical levels. Second, median daily concentrations of glycerol as a marker of cellular membrane degradation showed an increase one day after insulin onset, returning to baseline on day 4 .

In hours 9-12 of insulin infusion, a trend to a glycerol increase of unclear relevance had been observed previously (Schlenk et al 2008a). Regarding the long-term course, these glycerol changes might indicate a mild transient metabolic derangement following insulin infusion. However, the LPR remained stable and glutamate, a marker of neuronal injury and ischemia, decreased excluding ischemia as a relevant cause of cerebral glucose depletion and, even more important, indicating no substantial neuronal damage. Together, this pattern might be interpreted as absence of a relevant and continuous metabolic crisis following long-term insulin infusion, as long as cerebral glucose levels remain within the physiological range.

\section{Hyperglycemia}

Hyperglycemia at the time of brain injury, such as ischemic stroke or brain trauma, is known to be associated with increased morbidity and mortality (Walia et al 2002; Bhalla et al 2003). Persistence of hyperglycemia during the poststroke episode was found to be independently associated with infarct expansion and adverse outcome (Baird et al 2003). Preventive glycemic control with levels not substantially exceeding $6.1 \mathrm{mmol} / \mathrm{L}(110 \mathrm{mg} / \mathrm{dL})$ reduced mortality during intensive care by more than $40 \%$ (van den Berghe et al 2001). Nevertheless, thresholds for glucose levels to be treated with insulin vary considerably for SAH patients (van den Berghe et al 2001; Badjatia et al 2005; Frontera et al 2006). Since hyperglycemia appears to be injurious in brain ischemia conditions, avoidance of hyperglycemia should be a general strategy in treatment of SAH (Lanzino et al 


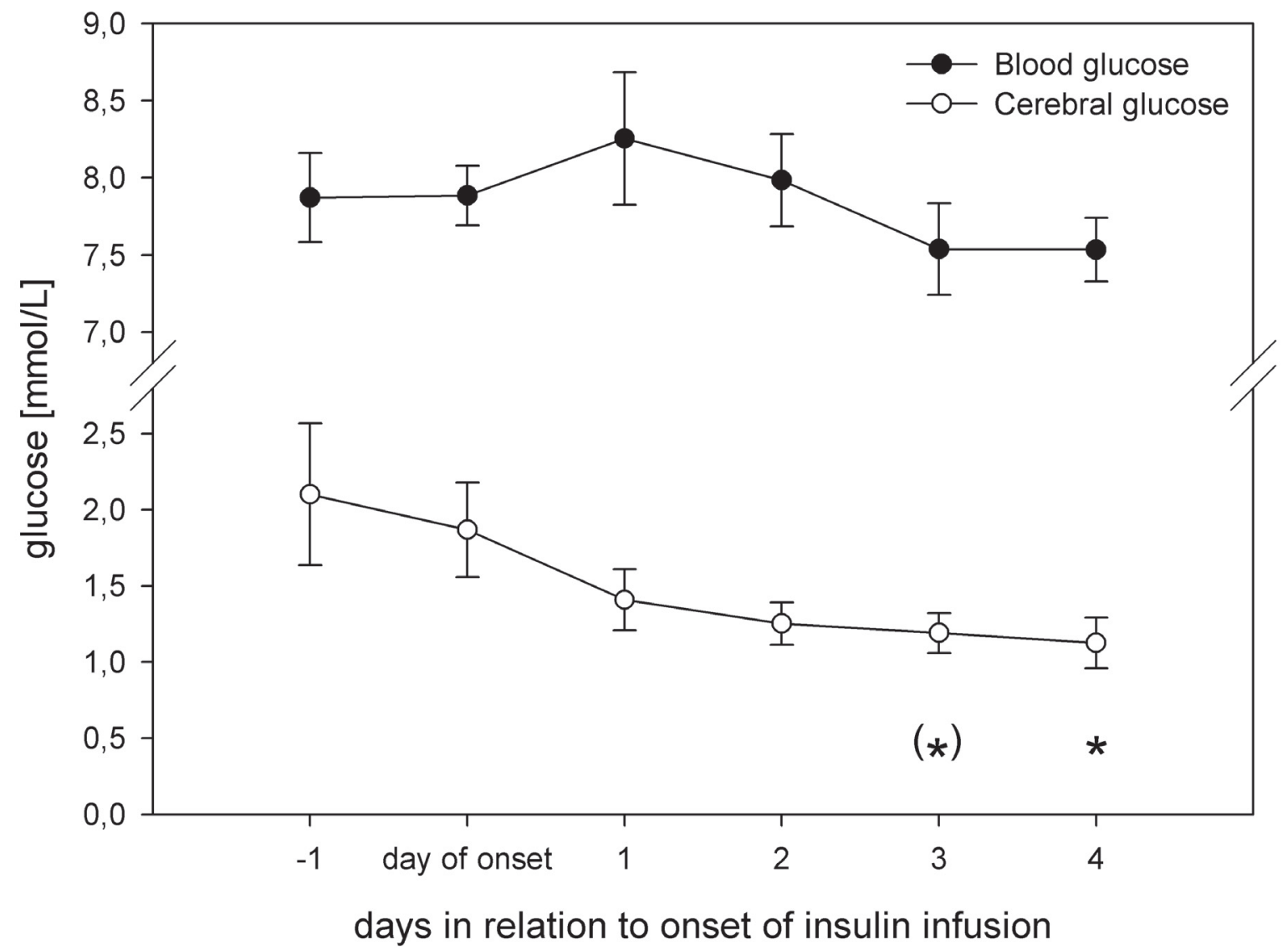

Figure I Course of plasma and cerebral glucose before and during insulin infusion. Data are expressed as mean \pm standard error of daily median values measured in 24 patients treated with continuous intravenous insulin. Levels of significance are indicated for comparison with glucose values on the day of insulin onset (Wilcoxon signedrank test).

Notes: ${ }^{*} \mathrm{p}<0.05,{ }^{(*)} \mathrm{p}<0.08$.

1993; Scott et al 1999; Sarrafzadeh et al 2004; Badjatia et al 2005; Frontera et al 2006). However, the cause-effect relationship between hyperglycemia and unfavorable outcome remains controversial as hyperglycemia might either occur stress-related and reflect - as a symptom - the severity of the primary injury, or act as a secondary force deteriorating brain damage and thereby contributing to adverse outcome. Furthermore, postulating an intact blood-brain barrier, high blood glucose does not necessarily imply high cerebral glucose levels. Several glucose transport proteins (GLUT) are involved in the delivery of glucose from the circulation to the brain, such as GLUT1 in glia and the microvascular endothelial cells forming the blood-brain barrier, and GLUT3 in neurons (Hamlin et al 2001).

Our results, though limited by various factors, indicate a significant insulin-related decrease in cerebral glucose levels though blood glucose remained stable. This lack of a significant decrease in blood glucose might be attributed to diverse factors such as catecholamines (either endogenous or iatrogenic administered) or the loose protocol for glycemic control rather accepting marginally elevated glucose levels than inducing hypoglycemia.

Since cerebral glucose remained within the physiological range even after the observed decrease, the complete recovery of the initial glycerol elevation and the lack of deterioration in the other markers of metabolic crisis (LPR and glutamate) are consistent. Future studies might detect relevant metabolic derangements when insulin is administered to patients with cerebral glucose levels already lowered in a critical way.

\section{Insulin and cerebral metabolism}

The debate about the role of insulin in cerebral glucose metabolism has not been resolved. In-vitro and experimental data demonstrated that insulin can penetrate the blood-brain barrier and enter the brain (Steffens et al 1988). The proof of insulin receptors in neuronal tissue raises the question of their functional significance (Kenner et al 1995). Recent data give evidence that physiologic levels of circulating insulin do not affect brain glucose uptake and that cerebral glucose uptake and metabolism is predominantly an 

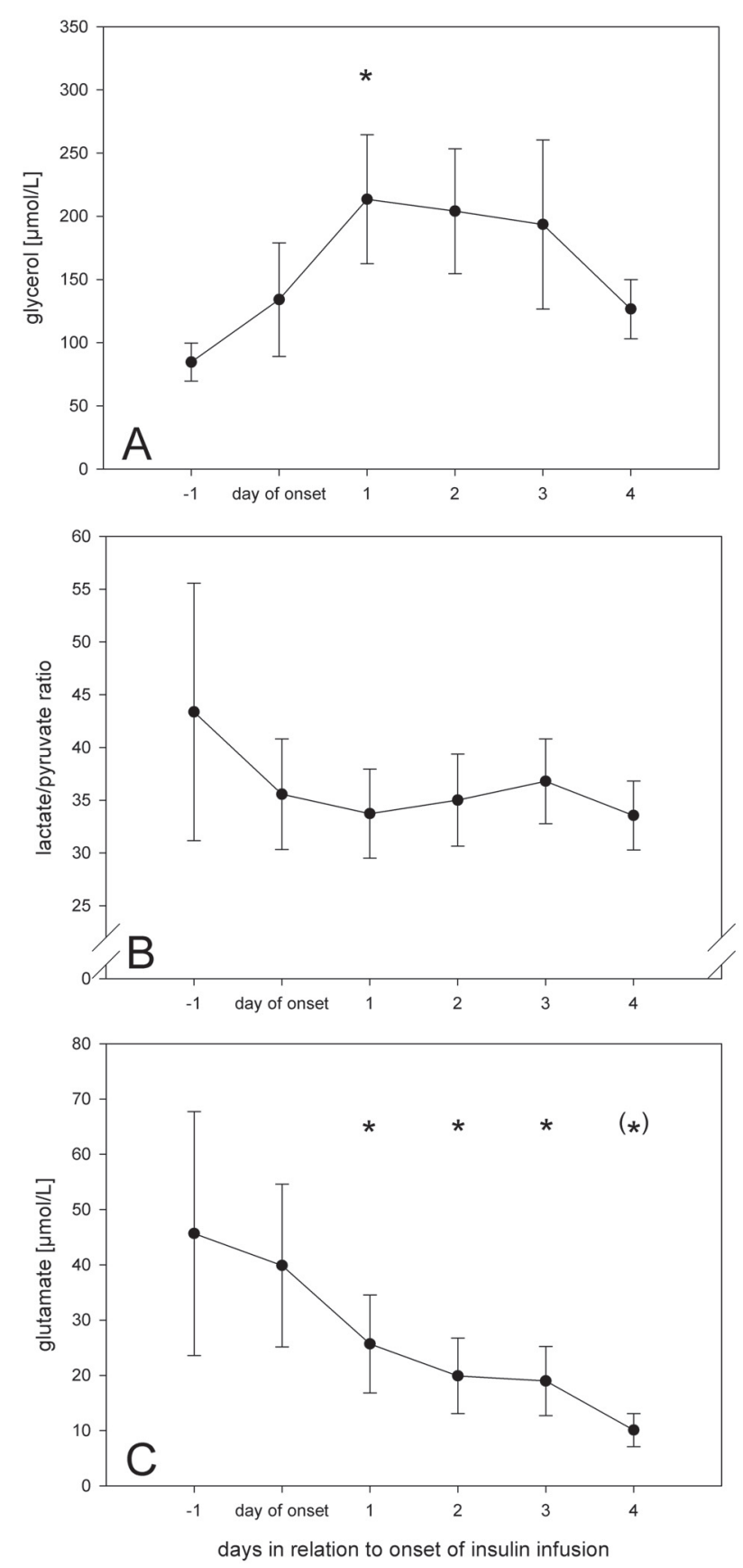

Figure 2 Course of markers of cerebral distress before and during insulin infusion (A) Glycerol,(B) Lactate/pyruvate ratio, (C) Glutamate. Data are expressed as mean \pm standard error of daily median values calculated from hourly measured microdialysate concentrations in 24 patients treated with continuous intravenous insulin. Levels of significance are indicated for comparison with microdialysate concentrations on the day of insulin onset (Wilcoxon signed-rank test).

Notes: ${ }^{p} \mathrm{p}<0.05,{ }^{(*)} \mathrm{p}<0.08$.

insulin-independent process in humans (Cranston et al 1998; Seaquist et al 2001). Nevertheless, data from TBI patients support the concern that a reduction in serum glucose could create substrate limitation in the injured brain (Vespa et al 2006). In the present study, insulin treatment appeared to be safe as median cerebral glucose concentrations did not fall below a critical threshold during insulin infusion. However, it is unclear whether insulin can be recommended in patients with low intracerebral glucose levels which might decrease further to critically low levels and compromise cerebral energy metabolism substantially. Therefore, in our view the monitoring of cerebral glucose especially at onset of and during insulin treatment is valuable in SAH patients. This might be relevant as a correlation between low cerebral glucose levels $(<0.2 \mathrm{mmol} / \mathrm{L})$ and unfavorable outcome could be shown in TBI patients (Vespa et al 2003).

\section{Current approaches for glycemic control in SAH}

The strategy of insulin treatment in this study was to rather tolerate marginally elevated blood glucose levels than to induce hypoglycemia. With the applied blood glucose target range (4.4-7.8 mmol/L; 80-140 mg/dL), no hypoglycemia but frequent hyperglycemia was observed. In a much-noticed publication on glucose control in critically ill patients, Van den Berghe suggests blood glucose levels between 4.4 and $6.1 \mathrm{mmol} / \mathrm{L}(80-110 \mathrm{mg} / \mathrm{dL})$, referring to data from cardiac surgery patients (van den Berghe et al 2001). A more recent publication, studying 63 patients with brain lesions (including brain tumors) demonstrated that intensive insulin therapy with blood glucose levels $<6.1 \mathrm{mmol} / \mathrm{L}(<110 \mathrm{mg} / \mathrm{dL})$ shortened intensive care dependency and reduced intracranial pressure as well as the need for vasopressors for maintaining adequate cerebral perfusion pressure (Van den Berghe et al 2005). It is questionable if these thresholds are applicable for patients with possibly compromised cerebral metabolism and regionally increased cerebral energy demand as described in aneurysmal SAH. Recently, the effect of intensive insulin therapy (4.4-6.7 mmol/L vs 4.4-12.2 $\mathrm{mmol} / \mathrm{L} ; 80-120 \mathrm{mg} / \mathrm{dL}$ vs $80-220 \mathrm{mg} / \mathrm{dL}$ ) was studied in 78 aneurysmal SAH patients, revealing that infection rates could be lowered by strict glycemic control, while a benefit concerning the incidence of DIND, outcome, and mortality was not found (Bilotta et al 2007).

\section{Limitations of this study}

The small and heterogenous study groups limit the conclusions that can be drawn concerning the effects of insulin, especially as patients with more severe hemorrhage are predominantly found in the insulin-treatment group. However, this was unavoidable as it seems not justifiable to exclude hyperglycemic patients from insulin treatment considering the strong evidence for an association between hyperglycemia 
and unfavorable outcome. Further, microdialysis is a regional method and the volume of brain tissue monitored by the microdialysis catheter covers only a few millimeters from the membrane. Some possibly serious effects of insulin on blood glucose might not have been detected because of the discontinuous blood glucose monitoring. To evaluate whether normalization of cerebral glucose levels can improve outcome in SAH patients, further studies are necessary.

\section{Conclusion}

This study confirms previous findings that hyperglycemia is a relevant complication in aneurysmal subarachnoid hemorrhage. Continuous intravenous insulin treatment over 4 days aiming at glucose levels $<7.8 \mathrm{mmol} / \mathrm{L}(<140 \mathrm{mg} / \mathrm{dL})$ was safe in respect to blood glucose as no hypoglycemia occurred. Insulin infusion induced a decrease in cerebral glucose on days 1-4 after onset while blood glucose levels and cerebral metabolism remained stable except for a reversible increase in glycerol indicating a transient metabolic derangement. However, to confirm the safety of insulin treatment in SAH, a larger study population is needed. In any case monitoring of blood and cerebral glucose appears to be valuable in SAH patients and may help to identify a deterioration of cerebral metabolism when insulin starts at critically low cerebral glucose levels.

\section{Disclosure}

The authors have no financial interest in the monitoring device described. The authors report no conflicts of interest.

\section{Acknowledgments}

The authors acknowledge the technical assistance of Sabine Seidlitz and Jasmin Kopetzki and the support of the ICU team, Charité Campus Virchow Medical Center, Germany.

\section{References}

Badjatia N, Topcuoglu MA, Buonanno FS, et al. 2005. Relationship between hyperglycemia and symptomatic vasospasm after subarachnoid hemorrhage. Crit Care Med, 33:1603-9.

Baird TA, Parsons MW, Phanh T, et al. 2003. Persistent poststroke hyperglycemia is independently associated with infarct expansion and worse clinical outcome. Stroke, 34:2208-14.

Bell DA, Strong AJ. 2005. Glucose/insulin infusions in the treatment of subarachnoid haemorrhage: a feasibility study. $\mathrm{Br} J$ Neurosurg, 19:21-4.

Bhalla A, Tilling K, Kolominsky-Rabas P, et al. 2003. Variation in the management of acute physiological parameters after ischaemic stroke: a European perspective. Eur J Neurol, 10:25-33.

Bilotta F, Spinelli A, Giovannini F, et al. 2007. The effect of intensive insulin therapy on infection rate, vasospasm, neurologic outcome, and mortality in neurointensive care unit after intracranial aneurysm clipping in patients with acute subarachnoid hemorrhage: a randomized prospective pilot trial. J Neurosurg Anesthesiol, 19:156-60.
Candelise L, Landi G, Orazio EN, et al. 1985. Prognostic significance of hyperglycemia in acute stroke. Arch Neurol, 42:661-3.

Cranston I, Marsden P, Matyka K, et al. 1998. Regional differences in cerebral blood flow and glucose utilization in diabetic man: the effect of insulin. J Cereb Blood Flow Metab, 18:130-40.

D’Alecy LG, Lundy EF, Barton KJ, et al. 1986. Dextrose containing intravenous fluid impairs outcome and increases death after eight minutes of cardiac arrest and resuscitation in dogs. Surgery, 100:505-11.

Frontera JA, Fernandez A, Claassen J, et al. 2006. Hyperglycemia after SAH: predictors, associated complications, and impact on outcome. Stroke, 37:199-203.

Ginsberg MD, Welsh FA, Budd WW. 1980. Deleterious effect of glucose pretreatment on recovery from diffuse cerebral ischemia in the cat. I. Local cerebral blood flow and glucose utilization. Stroke, 11:347-54.

Hamlin GP, Cernak I, Wixey JA, et al. 2001. Increased expression of neuronal glucose transporter 3 but not glial glucose transporter 1 following severe diffuse traumatic brain injury in rats. J Neurotrauma, 18:1011-8.

Hutchinson PJ, al-Rawi PG, O'Connell MT, et al. 2000a. On-line monitoring of substrate delivery and brain metabolism in head injury. Acta Neurochir Suppl, 76:431-5.

Hutchinson PJ, O'Connell MT, Al-Rawi PG, et al. 2000b. Clinical cerebral microdialysis: a methodological study. J Neurosurg, 93:37-43.

Hutchinson PJ, Gupta AK, Fryer TF, et al. 2002. Correlation between cerebral blood flow, substrate delivery, and metabolism in head injury: a combined microdialysis and triple oxygen positron emission tomography study. J Cereb Blood Flow Metab, 22:735-45.

Jennett B, Bond M. 1975. Assessment of outcome after severe brain damage. Lancet, 1:480-4.

Kenner KA, Kusari J, Heidenreich KA. 1995. cDNA sequence analysis of the human brain insulin receptor. Biochem Biophys Res Commun, 217:304-12.

Lanzino G, Kassell NF, Germanson T, et al. 1993. Plasma glucose levels and outcome after aneurysmal subarachnoid hemorrhage. J Neurosurg, 79:885-91.

Pulsinelli WA, Levy DE, Sigsbee B, et al. 1983. Increased damage after ischemic stroke in patients with hyperglycemia with or without established diabetes mellitus. Am J Med, 74:540-4.

Sarrafzadeh A, Haux D, Kuchler I, et al. 2004. Poor-grade aneurysmal subarachnoid hemorrhage: relationship of cerebral metabolism to outcome. J Neurosurg, 100:400-6.

Sarrafzadeh AS, Sakowitz OW, Kiening KL, et al. 2002. Bedside microdialysis: a tool to monitor cerebral metabolism in subarachnoid hemorrhage patients? Crit Care Med, 30:1062-70.

Saveland H, Nilsson OG, Boris-Moller F, et al. 1996. Intracerebral microdialysis of glutamate and aspartate in two vascular territories after aneurysmal subarachnoid hemorrhage. Neurosurgery, 38:12-9.

Schlenk F, Graetz D, Nagel A, et al. 2008a. Insulin-related decrease in cerebral glucose despite normoglycemia in aneurysmal subarachnoid hemorrhage. Crit Care, 12(1):R9.

Schlenk F, Nagel A, Graetz D, et al. 2008b. Hyperglycemia and cerebral glucose in aneurysmal subarachnoid hemorrhage. Intens Care Med, 34:1200-7.

Scott JF, Robinson GM, French JM, et al. 1999. Glucose potassium insulin infusions in the treatment of acute stroke patients with mild to moderate hyperglycemia: the Glucose Insulin in Stroke Trial (GIST). Stroke, 30:793-9.

Seaquist ER, Damberg GS, Tkac I, et al. 2001. The effect of insulin on in vivo cerebral glucose concentrations and rates of glucose transport/ metabolism in humans. Diabetes, 50:2203-9.

Steffens AB, Scheurink AJ, Porte D, Jr., et al. 1988. Penetration of peripheral glucose and insulin into cerebrospinal fluid in rats. Am J Physiol, 255:R200-4.

Ungerstedt U. 1991. Microdialysis - principles and applications for studies in animals and man. J Intern Med, 230:365-73.

van den Berghe G, Wouters P, Weekers F, et al. 2001. Intensive insulin therapy in the critically ill patients. N Engl J Med, 345:1359-67. 
Van den Berghe G, Schoonheydt K, Becx P, et al. 2005. Insulin therapy protects the central and peripheral nervous system of intensive care patients. Neurology, 64:1348-53.

Vespa P, Boonyaputthikul R, McArthur DL, et al. 2006. Intensive insulin therapy reduces microdialysis glucose values without altering glucose utilization or improving the lactate/pyruvate ratio after traumatic brain injury. Crit Care Med, 34:850-6.
Vespa PM, McArthur D, O'Phelan K, et al. 2003. Persistently low extracellular glucose correlates with poor outcome 6 months after human traumatic brain injury despite a lack of increased lactate: a microdialysis study. J Cereb Blood Flow Metab, 23:865-77.

Walia S, Sutcliffe AJ. 2002. The relationship between blood glucose, mean arterial pressure and outcome after severe head injury: an observational study. Injury, 33:339-44. 
